\title{
Revisiting the primary bias: the role of innumeracy in the misperception of prevalence of common illnesses
}

\section{Authors and affiliations:}

Jocelyn Raude ${ }^{1,2^{*}}$

Christina Xiao ${ }^{1}$

Pascal Crépey ${ }^{1,3}$

1) EHESP French School of Public Health, F-35000 Rennes, France ;

2) Unite des Virus Emergents (UVE: Aix-Marseille Univ - IRD 190 - Inserm 1207 - IHU Mediterranee Infection), Marseille, France;

3) Univ Rennes, EHESP, UMR CNRS 6051 Arènes, Rennes, France.

\section{*Corresponding author:}

Jocelyn Raude, PhD, Department of Social and Behavioral Sciences, EHESP French School of Public Health, 15 Avenue du Professeur Leon-Bernard, CS 74312, 35043 Rennes Cedex, France.

Tel.: +33299022615

Email: Џocelyn.Raude@ehesp.fr 


\section{Abstract:}

Although people have been repeatedly found to underestimate frequencies of common illnesses and overestimate those of rare illnesses, not much is known about this consistent bias in risk perception, termed "primary bias" in the literature, as well as the origin of its variations among different subpopulations. To fill this gap, we designed and implemented a national survey to compare the perceived and actual prevalence of common illnesses, and to test the hypothesis that numeracy may play an important role in the accuracy of judgments of risk frequencies. Our data were collected through a large online survey conducted in France among a representative sample of the adult population $(n=3,245)$. The participants were asked to complete a 10 -items numeracy scale and to estimate the prevalence of a variety of social conditions and common illnesses such as cancers or heart diseases in the French population by using a percentage scale. The analyses show that (1) participants tend to greatly overestimate the prevalence of conditions affecting small percentages of people, and underestimate those affecting a large percentage of them, (2) the Tversky and Kahneman's probability weighting function provides an adequate model to represent the discrepancy between the perceived and actual prevalence of these illnesses, and (3) the magnitude of the primary bias varies principally as a function of the respondents' numeracy. These results suggest that the primary bias that affects perceptions of prevalence of chronic diseases is not fundamentally different from those characterizing other types of probabilistic judgments investigated in the field of psychological and behavioral sciences. They also confirm that numeracy plays a considerable role in people's ability to transform epidemiological observations from their social environment into more accurate estimates.

Keywords: Primary bias, numeracy, risk judgments, chronic diseases, probability weighting function 


\section{Introduction}

Since the early stages of research on risk perception, scientists have shown through a number of famous surveys and laboratory experiments that people seem to display a range of recurrent and substantial biases when asked to think about the probability or frequency of health-related events (e.g., unrealistic optimism or conjunction fallacy). Notably, since the pioneering experimental research conducted by Attneave in the fifties then by Lichtenstein and her colleagues in the late seventies, it has been consistently found that people tend to underestimate the number of highfrequency events, and conversely, to overestimate that of low-frequency ones (Baron, 2006). In particular, it has been shown that the absolute frequencies of fatalities attributable to common chronic diseases (cancers, heart diseases, diabetes, etc.) were systematically underestimated, while those attributable to rare diseases or disasters - such as botulism or tornadoes - were greatly overestimated. This common phenomenon of distortion in the perception of risk, which is characterized by the flatness of the best-fit curve of the relationship between perceived and observed risks, has been called "primary bias" in the psychological literature (Gigerenzer et al., 2012; Johnson \& Tversky, 1983; Lichtenstein et al., 1978). For Lichtenstein et al (1978, p. 551), the primary bias refers to the common tendency "to overestimate small frequencies and overestimate larger ones". Nevertheless, it should be noted that at this stage the tendency to overestimate the low-frequency events and to underestimate the high-frequency events was not replicated in the study when their subjects had to rate the occurrence of events unrelated to health and illness such as the frequency of words or occupations.

During the same period, a series of experimental studies of decision-making under risk investigated probabilistic judgments by using more indirect elicitation methods based on choices between lotteries to infer potential distortions in the human perception of probability. The advantage of the indirect methods is that it they generally rely on more natural and less cognitively demanding tasks. This research has shown that subjective probabilities, or relative frequencies, are typically characterized by several remarkable mathematical properties (Budescu 
et al., 2011; Prelec, 1998; Tversky \& Kahneman, 1992). Firstly, probabilistic judgments can be represented as a regressive function, i.e., there is an inflexion value above which the subjective frequencies are lower than the objective frequencies of negative or positive events (and conversely). Secondly, probabilistic judgments can be represented by an asymmetrical curve, i.e., there exists a given value different from $50 \%$ at which the subjective frequencies equal the objective frequencies of events. As a consequence, the graphical representation of probabilistic judgments is represented as a sigmoid, or reverse S-shaped curve), i.e., the perceived risk frequencies are concave until the above-mentioned inflexion point, after which it is then convex. These empirical works have conducted to develop a model for the judgments related to probability in psychological and behavioral sciences using non-linear functions of transformation of objective probabilities, which show that the smaller frequencies tend to be overestimated and the larger frequencies are underestimated by subjects. According to Prelec (1998: 497), these subproportional probability weighting functions are generally "regressive, s-shaped and with a fixed point and invariant inflection point at $1 / e=.37 . "$

These research findings have nevertheless raised a number of criticisms among psychologists and behavioral scientists. First of all, most of the underlying data has been collected from small, convenient, and non-representative samples of undergraduate students from advantaged groups, which raises legitimate issues about the representativeness and generalizability of the data collected (Ioannidis \& Doucouliagos, 2013; Pashler \& Harris, 2012). However, it should be noted that a recent international study conducted in 19 countries successfully replicated most of the experimental findings underlying the prospect theory for decision under risk (Ruggeri et al., 2020). Second, some extensions of research devoted to primary bias in the area of health and illness have led to the collection of somewhat contradictory evidence (Raude et al., 2018). On one hand, the original finding by Lichtenstein et al (1978) that people tend to underestimate the higher absolute frequencies of deaths related to various biomedical causes (and overestimate the lower ones) has been successfully replicated by Hakes \& Viscusi (2004) among a non-representative sample of U.S. citizens, as well as by Hertwig et al. 
(2005) among a convenience sample of German students. However, other researchers found in larger samples of individuals that they were more likely to greatly overestimate some of the most common health threats, such as those associated with cigarette smoking (Lundborg \& Lindgren, 2004; Viscusi, 1990), diabetes or hypertension (Frijling et al., 2004). In other words, asking questions about the relative frequencies of harmful events, rather than their absolute frequencies, seems to lead to inconsistent or even contradictory results in risk perception research. These inconsistencies in the scientific evidence should incite us to develop an experimental design to test whether such biases in judgment of risk frequencies should be attributed to methodologicalexperimental artefacts or poor cognitive skills, including limited computational capacities (Gigerenzer, Fiedler, \& Olsson, 2012).

Thus, in this article, we took advantage of a large national health survey conducted in France to examine the conditions under which perceived prevalence of a variety of chronic illnesses is more or less biased (or inaccurate). The main research questions we wanted to address include the following: firstly, do the primary bias that has been observed in the aforementioned experiments on judgments of absolute frequency of an array of health-related risks also exist in a more ecologically realistic situation, in which people are asked to estimate the relative frequencies of widespread illnesses, and how reliable is the probability weighting model developed in the field of judgment and decision-making in predicting the perceived prevalence of these illnesses? Secondly, are the judgments of frequencies related to non-health events prone to the same bias as the judgment about the frequency of health events? Indeed, it seems that people are better at making frequency judgments about events they are more familiar with. For instance, Kahneman and Tversky (1973) found in their seminal research about base rate neglect that U.S. students made fairly accurate judgments when they were asked to estimate the proportion of graduate students in different academic fields. Thirdly, is the primary bias in frequency judgments specific to younger and more educated subjects or are they potentially universal across social groups? Given the extensive body of literature that exists on the socioeconomic and demographic variations in both numerical and non-numerical judgments about health risks 
(Finucane et al., 2000; Hakes \& Viscusi, 2004; Kahan et al., 2007), it seems reasonable to assume that there may be considerable differences among people and groups in the accuracy of their estimates of illness prevalence. Fourthly, can these potential sociodemographic variations in the accuracy of frequency judgment be accounted for by the unequal distribution of numeracy skills within the whole population? Indeed, there is now growing evidence that individuals with higher numerical ability exhibit much less biased perceptions of health risk and benefit than those with poorer numerical ability (for a review, see Reyna et al., 2009).

\section{Subjects and Methods}

The data were collected from an online survey of French adults (18 years or older) participating in a large national panel devoted to research on consumer attitudes and behaviors. The panelists consented to participate in surveys on a regular basis in exchange for financial compensation. For the sake of representativeness, the panel included individuals who were recruited in both online and offline populations. However, only online panelists were offered to participate, as telephone interviews were not suitable for the type of questions submitted to the subjects. The survey complied with the recommendations of the National Data Protection Authority (CNIL), which is responsible for the ethical issue and protection of personal data collected in France. All subjects were informed about the objective of the research and gave electronic informed consent. In total, 6,000 panelists were contacted by email in October of 2014, and 3,245 agreed to respond to the survey (cooperation rate $=54 \%$ ).

\section{Measures}

Observed prevalence of chronic illnesses: For this study, we selected a list of 12 common chronic illnesses, i.e. "conditions that last a year or more and require ongoing medical attention and/or limit activities of daily living" (Bernell \& Howard, 2016). This includes communicable such as infectious diseases (e.g., HIV or hepatitis), non-communicable illnesses (e.g., cancers or heart diseases), and metabolic and endocrine disorders (e.g., diabetes, high blood sugar). Statistics 
provided by the national public health authorities in their annual report on the state of the population's health were used to measure the objective prevalence of a variety of chronic diseases in France (DREES, 2015). As shown in Table 1, the actual prevalence of these illnesses in the French population ranged from a rate of $1.1 \%$ for genetic disorders to $57.8 \%$ for eye diseases such as myopia or astigmatism.

Perceived prevalence of chronic illnesses: To date, there is still a lot of disagreement among scientists on how judgments of risk frequencies should be measured in empirical research (Weinstein, 1998). As noted by Viscusi (1990, p. 1256): “Obtaining meaningful survey responses regarding individuals' risk perceptions is not a straightforward task". This methodological problem can also be attributed to the fact that elicitation methods are more or less natural and often require considerable cognitive efforts from individuals. Furthermore, there exist substantial variations in the definition and measurement of the concept of risk among scientific disciplines (Althaus, 2005). For instance, epidemiologists commonly assess health risk by calculating the incidence (rate of new cases within a given time frame) or prevalence (proportion of cumulative cases at a certain time point) of an illness or disability in a given population. This information about the relative frequency of a disease can alternatively be expressed in terms of fractions, odds, or percentages. However, people's comprehension of these various numerical expressions of how common a disease is within a population can be taken for granted since eliciting probabilistic judgments has been repeatedly found to pose a number of difficulties in research on risk perception (Diefenbach et al., 1993; Gigerenzer, 1991; Peters, 2008; Rothman \& Kiviniemi, 1999).

To address this issue, participants were asked to estimate the prevalence of the 12 abovementioned chronic illnesses (see Table 1), by using a percentage scale, i.e., a relative frequency scale based on a reference sample of 100 individuals. Although subjects in laboratory experiments have been shown to have some difficulties using and understanding numerical information, there is sound evidence that using a more succinct percentage format is a better 
method than using other numeric formats to represent simple probabilities (Woloshin \& Schwartz, 2011). In our survey, the format and phrasing of the questions were adapted from the item developed by Viscusi (1990): “Among 100 people living in France, how many do you think have (Disease)? Please give a value between 0 and 100". Thus, the accuracy of people's judgments of the percentages measured through this method provides insight into the accuracy of people's judgments on the relative frequency of specific illnesses, or the perceived prevalence, by comparing them with the estimates of "objective" prevalence based on epidemiological data (the observed prevalence).

Perceived and actual prevalence of occupational status: In the same vein, the participants were asked to estimate the proportion in the French population of a series of 13 occupational and employment statuses, such as the current unemployment or retirement rates. The format and phrasing of the questions were identical to those employed to estimate the prevalence of specific illnesses: “Among 100 people living in France, how many do you think are (Occupation)? Please give a value between 0 and 100". The data used to determine the objective prevalence of these occupational and employment statuses in the French population were drawn from the annual statistics released in 2015 by the French national institute for statistical and economic studies (INSEE).

Numeracy: Numeracy is generally defined as the ability to comprehend and use simple mathematical and probabilistic concepts (Peters, 2008). To date, numerous numeracy scales have been proposed in the literature to test the objective ability of individuals to understand and/or compute numbers (Reyna et al, 2009). Here, the participants were asked to complete a 10 -items numeracy scale developed by Lipkus et al. (2001) to measure each subject's ability to deal with numeric information about risks and benefits, e.g., "Imagine that we flip a fair coin 1,000 times. What is your best guess about how many times the coin would come up heads in 1,000 flips?", and "Which of the following represents the biggest risk of getting a disease? 1\%, 10\%, 5\%?". This scale showed a relatively high internal consistency (Cronbach's $\alpha=.77$ ). Thus, a numeracy score 
could be calculated for each participant by summing the total number of correct responses for these 10 items.

Socioeconomic and demographic characteristics: A variety of sociodemographic and health characteristics were systematically collected among the participants at the beginning of the survey. These included gender, sex, age, level of education, occupation, household income, location, housing conditions, family and marital status, as well as perceived health status. The characteristics of the sample are shown in Table 1.

\section{Statistical Analysis}

We calculated arithmetic means and 95\% confidence intervals for the perceived relative frequencies of each socioeconomic and illness-related variables. We used t-tests to compare statistical differences in means among various categories of respondents. We examined the relationship between the perceived and observed prevalence of diseases or socio-occupational status by using a probability weighting model developed in the field of behavioral decisionmaking (Prelec, 1998). Behavioral studies of judgment and decision-making under risk have demonstrated through a series of laboratory experiments that the relationship between subjective and objective probabilities of events can be adequately described by a nonlinear transformation of the relative frequencies defined as:

$w(p)=\exp \left(-\beta(-\ln (p))^{\alpha}\right.$ where $\alpha$ and $\beta \in[0,1]$ and $p$ is the actual probability or frequency.

This weighting function implies that there exists an inflection point under which the deviation between the perceived and observed prevalence gets smaller as the observed prevalence increases (and vice versa). A simple analysis of this function shows that the magnitude of the deviance is controlled by $\alpha$ and the position of the inflection point by $\beta$. Hence, we can estimate $\alpha$ on subsets of the population to measure how a specific characteristic impacts the curvature of the perception curve. Our estimation procedure relies on the box-constrained version of the BFGS optimization method (Byrd et al., 1995), as implemented in the package 'optimx', to estimate $\alpha$ 
and $\beta$ by minimizing the mean squared error (MSE) of $w(p)$. Other optimization methods have been tested and gave similar or higher MSE. All the analyses were performed using R 3.6.1 (with R Studio 1.2).

We also wanted to perform multivariate analysis of the potential factors associated with the deviation between a perceived and an observed prevalence. To that purpose we considered the following equation for $\alpha$ :

$$
\alpha=\frac{1}{1+\exp (-\gamma)}
$$

so that $\alpha \in[0,1]$ and where we define $\gamma$ has

$$
\gamma=\sum \delta_{i} X_{i}+\epsilon
$$

where $X_{i}$ are the studied cofactors and $\delta_{i}$ their estimated coefficients.

We used the same estimation procedure to estimate $\delta_{i}$ and $\beta$. We computed the determination coefficient $R^{2}$ as a measure of fitness quality, and produced $95 \%$ confidence intervals of the estimates from the numerically estimated Hessian matrix.

To quantify the impact of cofactor $i$ on the perception curve, we defined the Mean Error Increase (MEI) as:

$$
M E I_{i}=\frac{E\left(\left|P-w\left(p, X_{i}=1, X_{j \neq i}=0\right)\right|\right)-E(|P-w(p, X=0)|)}{E(|P-w(p, X=0)|)}
$$

where $P$ is the observed prevalence and $X$ are defined as dichotomous variables whose reference value is $0 . M E I_{i}$ measures the proportion of increase (or decrease) in the perception error depending solely on $X_{i}$ when all other cofactors are at their reference value. We also define the Mean Absolute Error (MAE) and the Mean Relative Error as:

$$
M A E_{i}=E\left(\left|P-w\left(p, X_{i}=1, X_{j \neq i}=0\right)\right|\right)
$$

and

$$
M R E_{i}=E\left(\left|P-w\left(p, X_{i}=1, X_{j \neq i}=0\right)\right| / P\right)
$$


$M A E_{i}$ and $M R E_{i}$ measure the average absolute or relative difference (respectively) between the perceived and observed prevalence for each covariate included in the analysis when all others are at their reference value.

\section{Results}

\section{Are the perceived prevalence of common chronic illnesses biased and how?}

As shown in Table 1, the participants tend to significantly overestimate a large majority of the most common illnesses in France $(t<4.4, d f<3,245, p<.001)$. However, the nature and magnitude of this bias in the perception of the prevalence of these illnesses showed substantial differences, which depend to a large extent on their actual prevalence. First, the discrepancy between the perceived and actual prevalence calculated for each illness was considerably higher for the less prevalent illnesses than for the more prevalent illnesses in the population (e.g., the prevalence of infectious diseases and genetic disorders are overestimated by about a factor of 10 and 12 , respectively, while that of metabolic disorders was only overestimated by $20 \%$ ). Second, the overestimation bias observed in the prevalence of these illnesses tends to decrease until a certain threshold, which represents the correct estimation, then switches into an underestimation that increases as the magnitude of the prevalence increases. In other words, there exists a phenomenon of overestimation when prevalence is low, while there is one of underestimation when prevalence is high. In addition, it should be noted that contrary to the results obtained by Lichtenstein and her colleagues, the same pattern was found in our study for the estimates of the rate of a variety of occupational and employment statuses in the French population.

Overall, participants tended to provide more accurate or unbiased estimates when the actual prevalence rate of the status was closer to the inflection point (1/e) defined by Prelec. This suggests that the primary bias that affects perceptions of prevalence of chronic diseases may not be fundamentally different from those characterizing other types of frequency or probability 
judgments. Last but not least, we found that the Tversky and Kahneman's probability weighting function provided a suitable mathematical model for this primary bias in the perceived prevalence of common illnesses.

\section{Does the 'primary' bias vary in magnitude as a function of individual characteristics?}

In line with the results of previous studies, the magnitude of the primary bias in the perceived prevalence of common illnesses appears to vary as a function of the participants' sex, level of education and numeracy. As shown in Figures 1 and 2 (for the details, see Table 2), the calculated alpha coefficients, which represents the slope of the reversed S-shaped curve at the infection point, were 0.56 for males versus 0.37 for females, and 0.54 for the more educated versus 0.30 for the less educated participants. Furthermore, the discrepancy between the perceived and the actual prevalence of chronic diseases was found to vary to a lesser extent by age group, occupation and health status, and to a larger extent according to the individual's level of numeracy. As shown in Figure 3, the alpha coefficient was 0.63 for the more numerate versus 0.28 for the less numerate. However, as shown in Figure 4, there exists significant variations in the participants' numeracy score depending on their $\operatorname{sex}(t(3,243)=7.9, p<.001)$ and level of education $(F(3,244)=219.1, p<.001)$.

The multivariate analysis, including all studied cofactors, allowed us to account for all covariates at the same time, hence removing potential confounding effects (Table 3). The determination coefficient $\left(R^{2}\right)$ of the multivariate model was $18 \%$ and covariates whose MEI 95\% confidence interval including $0 \%$ were considered non-statistically significant. In this analysis, a low numeracy index accounted for 59.3\% (MEI, 95\% CI [43.6\%; 74.2\%]) of the primary bias estimated by the model, which may lead to a relative deviation of the perceived prevalence of 105.4\% (MRE, 95\%CI [75.4\% ; 146.3\%]). The sociodemographic factors were also found to play an important role in the misperception of the prevalence as the sex of the participants accounted for $47.9 \%$ (95\%CI $[32.7 \% ; 68.1 \%]$ ) of the primary bias, and a low education level (limited to middle school) and being young (18 to 39 years old) accounted for 18.7\% (95\%CI [11.5\%; 
$27.0 \%]$ ) and $11.6 \%$ (95\%CI [3.0\%; 21.9\%]), respectively. The other socio-demographic covariates and the type of estimate (common illnesses versus occupations) did not significantly affect the magnitude of the primary bias.

\section{Is the effect of sex and education on the primary bias mediated by numeracy?}

To determine whether sex and educational differences in the magnitude of the primary bias were due to differences in numerical abilities (see fig. 4), we used methods developed by Preacher and Hayes (2008) which allowed us to estimate path coefficients in simple and multiple mediator models and to provide confidence intervals for both direct and indirect effects. In this mediation model, sex and education were the independent variables; the level of numeracy was the mediator, and the alpha coefficient, which represents the slope of the probability weighting function at the inflexion point, was the dependent variable. The Hayes and Preacher's macro (2011) was used to calculate the parameter estimates and bootstrap confidence intervals. The results are shown in Figure 5. Interestingly, sex and education still had both a significant direct effect on the magnitude of the primary bias after adjusting for the indirect effects of numeracy ( $p$ $<.001)$. The people's numerical ability accounted for $45 \%$ of the relationship between the level of formal education and numeracy, but only $21 \%$ of the relationship between sex and numeracy. Thus, the gender gap in the accuracy of the perceived prevalence of illnesses was not mostly explained by the level of numeracy.

\section{Discussion}

How accurate are judgments that people make about risk frequencies? Despite the fact that perceptions of risk have been extensively studied since the famous works conducted by Lichtenstein and colleagues, there is still pervasive disagreement among psychologists and behavioral scientists about people's capabilities to estimate frequencies and numbers related to health and illness in an unbiased manner. On one hand, a number of studies consistently showed that people were likely to overestimate rare health risks (and underestimate common health 
risks), a psychological effect named 'primary bias' in the literature about judgement and decisionmaking (Baron, 2006). On the other hand, the overestimation or underestimation of certain health risks seems to depend, to a large extent, to the type of elicitation methods solicited by the researchers to examine risk judgements (Weinstein, 1998). According to Fischhoff ( 2012, p. 188), "systematic research on this question (how accurate are lay perception of risk) has only just begun". Although Fischhoff's opinion about the state of current knowledge regarding the perception of health risks may be viewed as overly pessimistic, it nevertheless points out a potential lack of replication and refutation culture in this field. Notably, since the seminal work conducted by Tversky and Kahneman in the seventies, questions about whether some common cognitive biases in risk-related judgment and decision-making persist across a variety of populations and communities has not been seriously considered and reinvestigated until only recently (Ruggeri et al, 2020).

In this study, we tested the hypothesis that the Tversky and Kahneman's probability weighting function accounts for the primary bias in risk judgements in ecologically realistic conditions. In line with the results from a previous study of risk judgements related to infectious diseases (Raude et al, 2018), we found that the probability weighting function provided a suitable mathematical model for this bias in the perceived prevalence of common illnesses. This is a remarkable finding as this function was initially developed by Tversky and Kahneman on the basis of an indirect elicitation method, which led them to infer the reversed S-shape of probabilistic judgments from a series of choices under risk made by the subjects enrolled in experimental studies. Nevertheless, even though the perceptions of risk have long been recognized as an important variable within the leading theories of human behaviors, we still know relatively little about the social and individual variations - and therefore the origins - of some well-known cognitive biases, which shape the way people think about the probability or frequency of negative events. For instance, due to the large utilization of small and convenient samples in the past research, it should be noted that most studies did not investigate the role of demographic factors such as nationality, gender or education, in the accuracy of risk perceptions 
(Hakes \& Viscusi, 2004; Raude et al., 2005). Therefore, we still do not know whether the primary bias observed in the judgments of probability or frequency are either potentially universal or more specific to certain categories of people.

Moreover, to the best of our knowledge, no study has investigated the effect of numeracy on the magnitude of the primary bias in risk judgements. This is relatively surprising as (1) a number of studies about cognitive biases in risk perception have shown consistent differences among subgroups of population according to their numerical skills, and (2) the level of numeracy was repeatedly found to vary as a function of the respondents' gender, socioeconomic status, or education (Peters, 2008; Reyna et al., 2009). Notably, a large majority of the studies examining the role of innumeracy in the misperception of health-related risks has been conducted in relation to breast cancer. Most of them came to the conclusion that people are likely to overestimate their personal risk of developing the disease when compared to the epidemiological evidence, with the less numerate participants providing more biased risk estimates than the more numerate ones. In other words, the misperception of risks related to breast cancer seems to depend to a large extent on the level of innumeracy. For instance, Woloshin and his colleagues found that women lower in numeracy provided more inaccurate risk estimates than their counterparts higher in numeracy (Schwartz et al., 1997). Similarly, Davids et al found that all women overestimated their risk of dying from breast cancer. However, participants exhibiting higher level of numeracy appeared to make significantly less biased risk judgments than those with poor numerical abilities (Davids et al., 2004).

Finally, our study confirms that accuracy of risk perceptions varies largely according to people's numerical skills, which are unequally distributed across sociodemographic groups. Consistent with past research, better educated and male participants have a higher level of numeracy. Overall, individuals lower in numeracy tend to systematically overestimate the prevalence of conditions affecting less than $20-35 \%$ of the population and to underestimate that of more common conditions, such as eyes disorders and diseases, when compared to those higher in 
numeracy. Furthermore, we found that people's numerical skills accounted to a large extent for the variations observed in past research in the magnitude of the primary bias in risk judgements as a function of the socioeconomic and demographic characteristic of the participants (Hakes \& Viscusi, 2004, Raude et al, 2018).

\section{Conclusion}

By revisiting the primary bias discovered by Lichtenstein and colleagues in the late seventies, this study contributes significantly, in our opinion, to a better understanding of this phenomenon. First, it shows that the weighting probability function provides an adequate model to account for this well-documented bias in the perceived prevalence of common illnesses, as well as other personal conditions or characteristics such as occupations. Second, it shows how this bias varies across populations, with a lack of basic numerical skills playing an essential role in the inaccuracy of the perceived prevalence of common illnesses. Indeed, most people did not express unbiased judgments but innumeracy appears to substantially increase the misperception of health-related risks. Third, these results indicate that numeracy not only reflects the people's ability to understand and use statistical information in their decision-making, but also their ability to transform routine observations and discussions about cases of illness in personal networks and public sources of information, such as television and social media, in more accurate health risk estimates. Last but not least, they suggest that certain common biases in frequency judgments may be considerably reduced by education and practice.

\section{References}

Althaus, C. E. (2005). A Disciplinary Perspective on the Epistemological Status of Risk. Risk Analysis, 25(3), 567-588. https://doi.org/10.1111/j.1539-6924.2005.00625.x

Baron, J. (2006). Thinking and Deciding (4e éd.). Cambridge University Press. https://doi.org/10.1017/CB09780511840265 
Bernell, S., \& Howard, S. W. (2016). Use your words carefully : What is a chronic disease? Frontiers in public health, 4, 159.

Budescu, D., Abbas, A., \& Wu, L. (2011). Does probability weighting matter in probability elicitation? Journal of Mathematical Psychology, 55(4), 320-327. https://doi.org/10.1016/j.jmp.2011.04.002

Byrd, R. H., Lu, P., Nocedal, J., \& Zhu, C. (1995). A Limited Memory Algorithm for Bound Constrained Optimization. SIAM Journal on Scientific Computing, 16(5), 1190-1208. https://doi.org/10.1137/0916069

Davids, S. L., Schapira, M. M., McAuliffe, T. L., \& Nattinger, A. B. (2004). Predictors of pessimistic breast cancer risk perceptions in a primary care population. Journal of General Internal Medicine, 19(4), 310-315. https://doi.org/10.1111/j.1525-1497.2004.20801.x

Diefenbach, M. A., Weinstein, N. D., \& O’Reilly, J. (1993). Scales for assessing perceptions of health hazard susceptibility. Health Education Research, 8(2), 181-192. https://doi.org/10.1093/her/8.2.181

DREES. (2015). L'état de santé de la population en France-Rapport 2015. Direction de la recherche, des études, de l'évaluation et des statistiques. https://drees.solidaritessante.gouv.fr/publications/rapports/letat-de-sante-de-la-population-en-france-rapport2015

Finucane, M. L., Slovic, P., Mertz, C. K., Flynn, J., \& Satterfield, T. A. (2000). Gender, race, and perceived risk : The « white male » effect. Health, Risk \& Society, 2(2), 159-172. https://doi.org/10.1080/713670162

Fischhoff, B. (2012). Communicating Risks and Benefits : An Evidence Based User's Guide. Government Printing Office.

Frijling, B. D., Lobo, C. M., Keus, I. M., Jenks, K. M., Akkermans, R. P., Hulscher, M. E. J. L., Prins, A., van der Wouden, J. C., \& Grol, R. P. T. M. (2004). Perceptions of cardiovascular risk among patients with hypertension or diabetes. Patient Education and Counseling, 52(1), 47-53. https://doi.org/10.1016/S0738-3991(02)00248-3 
Gigerenzer, G. (1991). How to make cognitive illusions disappear : Beyond "heuristics and biases". European review of social psychology, 2(1), 83-115.

Gigerenzer, G., Fiedler, K., \& Olsson, H. (2012). Rethinking cognitive biases as environmental consequences. Ecological rationality: Intelligence in the world, 80-110.

Hakes, J. K., \& Viscusi, W. K. (2004). Dead Reckoning : Demographic Determinants of the Accuracy of Mortality Risk Perceptions. Risk Analysis, 24(3), 651-664. https://doi.org/10.1111/j.0272-4332.2004.00465.x

Hayes, A., \& Preacher, K. (2011). Multicategorical X in Mediation Analysis Indirect and Direct Effects of a Multicategorical Causal Agent in Statistical Mediation Analysis.

Hertwig, R., Pachur, T., \& Kurzenhäuser, S. (2005). Judgments of Risk Frequencies : Tests of Possible Cognitive Mechanisms. Journal of Experimental Psychology: Learning, Memory, and Cognition, 31(4), 621-642. https://doi.org/10.1037/0278-7393.31.4.621

Ioannidis, J., \& Doucouliagos, C. (2013). What's to Know About the Credibility of Empirical Economics? Journal of Economic Surveys, 27(5), 997-1004. https://doi.org/10.1111/joes.12032

Johnson, E. J., \& Tversky, A. (1983). Affect, generalization, and the perception of risk. Journal of Personality and Social Psychology, 45(1), 20-31. https://doi.org/10.1037/00223514.45.1.20

Kahan, D. M., Braman, D., Gastil, J., Slovic, P., \& Mertz, C. K. (2007). Culture and IdentityProtective Cognition : Explaining the White-Male Effect in Risk Perception. Journal of Empirical Legal Studies, 4(3), 465-505. https://doi.org/10.1111/j.17401461.2007.00097.x

Kahneman, D., \& Tversky, A. (1973). On the psychology of prediction. Psychological Review, 80(4), 237-251. https://doi.org/10.1037/h0034747

Lichtenstein, S., Slovic, P., Fischhoff, B., Layman, M., \& Combs, B. (1978). Judged frequency of lethal events. Journal of Experimental Psychology: Human Learning and Memory, 4(6), 551-578. https://doi.org/10.1037/0278-7393.4.6.551 
Lipkus, I. M., Samsa, G., \& Rimer, B. K. (2001). General performance on a numeracy scale among highly educated samples. Medical decision making, 21(1), 37-44.

Lundborg, P., \& Lindgren, B. (2004). Do They Know What They are Doing? Risk Perceptions and Smoking Behaviour Among Swedish Teenagers. Journal of Risk and Uncertainty, 28(3), 261-286. https://doi.org/10.1023/B:RISK.0000026098.84109.62

Pashler, H., \& Harris, C. R. (2012). Is the Replicability Crisis Overblown? Three Arguments Examined. Perspectives on Psychological Science, 7(6), 531-536. https://doi.org/10.1177/1745691612463401

Peters, E. (2008). Numeracy and the perception and communication of risk. Annals of the New York Academy of Sciences, 1128(1), 1-7.

Prelec, D. (1998). The Probability Weighting Function. Econometrica, 66(3), 497-527. https://doi.org/10.2307/2998573

Raude, J., Fischler, C., Setbon, M., \& Flahault, A. (2005). Scientist and public responses to BSErelated risk : A comparative study. Journal of Risk Research, 8(7-8), 663-678.

Raude, J., Peretti-Watel, P., Ward, J., Flamand, C., \& Verger, P. (2018). Are Perceived Prevalences of Infection also Biased and How? Lessons from Large Epidemics of Mosquito-Borne Diseases in Tropical Regions. Medical Decision Making, 38(3), 377-389. https://doi.org/10.1177/0272989X17750845

Reyna, V. F., Nelson, W. L., Han, P. K., \& Dieckmann, N. F. (2009). How numeracy influences risk comprehension and medical decision making. Psychological Bulletin, 135(6), 943-973. https://doi.org/10.1037/a0017327

Rothman, A. J., \& Kiviniemi, M. T. (1999). Treating People With Information : An Analysis and Review of Approaches to Communicating Health Risk Information. JNCI Monographs, 1999(25), 44-51. https://doi.org/10.1093/oxfordjournals.jncimonographs.a024207

Ruggeri, K., Alí, S., Berge, M. L., Bertoldo, G., Bjørndal, L. D., Cortijos-Bernabeu, A., Davison, C., Demić, E., Esteban-Serna, C., \& Friedemann, M. (2020). Replicating patterns of prospect theory for decision under risk. Nature human behaviour, 4(6), 622-633. 
Schwartz, L. M., Woloshin, S., Black, W. C., \& Welch, H. G. (1997). The Role of Numeracy in Understanding the Benefit of Screening Mammography. Annals of Internal Medicine, 127(11), 966-972. https://doi.org/10.7326/0003-4819-127-11-199712010-00003

Tversky, A., \& Kahneman, D. (1992). Advances in prospect theory: Cumulative representation of uncertainty. Journal of Risk and Uncertainty, 5(4), 297-323.

https://doi.org/10.1007/BF00122574

Viscusi, W. K. (1990). Do Smokers Underestimate Risks? Journal of Political Economy, 98(6), 1253-1269. https://doi.org/10.1086/261733

Weinstein, N. D. (1998). Accuracy of smokers' risk perceptions. Annals of Behavioral Medicine, 20(2), 135-140. https://doi.org/10.1007/BF02884459

Woloshin, S., \& Schwartz, L. M. (2011). Communicating data about the benefits and harms of treatment : A randomized trial. Annals of internal medicine, 155(2), 87-96. 
Table 1. Difference between perceived and observed prevalence of chronic diseases and occupational status in France: actual percentage, mean estimated percentage, difference in percentage points (standard deviation), t-value ( $p$-value), and ratio.

\begin{tabular}{|c|c|c|c|c|c|}
\hline Variable & $\begin{array}{l}\text { Actual } \\
\text { prevalence }\end{array}$ & $\begin{array}{l}\text { Mean perceived } \\
\text { prevalence }[95 \% \mathrm{Cl}]\end{array}$ & $\begin{array}{l}\text { Difference } \\
\text { between perceived } \\
\text { and actual } \\
\text { prevalence (SD) }\end{array}$ & t-value ( $p$-value) & $\begin{array}{l}\text { Ratio perceived / } \\
\text { actual prevalence }\end{array}$ \\
\hline Eye diseases & $57.8 \%$ & $42.6 \%[41.8 \% ; 43.5 \%]$ & $-0.15(0.24)$ & $-34.4(<0.001)$ & 0.738 \\
\hline Working population & $51.2 \%$ & $48.2 \%[47.5 \% ; 48.9 \%]$ & $-0.03(0.18)$ & $-8.73(<0.001)$ & 0.942 \\
\hline Retired population & $31.9 \%$ & $32.1 \%[31.4 \% ; 32.8 \%]$ & $0(0.18)$ & $0.62(0.53)$ & 1.007 \\
\hline $\begin{array}{l}\text { Administration/Teaching/ } \\
\text { Health workers }\end{array}$ & $30.3 \%$ & $27.4 \%$ [26.8\%;28.1\%] & $-0.03(0.18)$ & $-8.65(<0.001)$ & 0.906 \\
\hline Cardiovascular diseases & $28.2 \%$ & $25.9 \%[25.2 \% ; 26.6 \%]$ & $-0.02(0.2)$ & $-6.31(<0.001)$ & 0.918 \\
\hline $\begin{array}{l}\text { Nutritional \& metabolic } \\
\text { diseases }\end{array}$ & $25.6 \%$ & $30.4 \%[29.6 \% ; 31.2 \%]$ & $0.05(0.21)$ & $12.17(<0.001)$ & 1.188 \\
\hline Respiratory diseases & $20.6 \%$ & $28.6 \%[27.8 \% ; 29.4 \%]$ & $0.08(0.21)$ & $20.44(<0.001)$ & 1.389 \\
\hline Digestive diseases & $15.4 \%$ & $20.9 \%[20.2 \% ; 21.6 \%]$ & $0.05(0.19)$ & $15.53(<0.001)$ & 1.355 \\
\hline Mental illnesses & $14.0 \%$ & $15.1 \%[14.5 \% ; 15.7 \%]$ & $0.01(0.16)$ & $3.47(<0.001)$ & 1.076 \\
\hline Manufacturing workers & $13.5 \%$ & $22.7 \%[22.1 \% ; 23.3 \%]$ & $0.09(0.17)$ & $29.8(<0.001)$ & 1.682 \\
\hline Sales workers & $12.4 \%$ & $25.3 \%$ [24.5\%;26\%] & $0.13(0.19)$ & $35.74(<0.001)$ & 2.036 \\
\hline Neurologic disorder & $10.8 \%$ & $20.4 \%$ [19.7\%;21.1\%] & $0.1(0.19)$ & $27.48(<0.001)$ & 1.890 \\
\hline Skin diseases & $9.7 \%$ & $24.1 \%[23.4 \% ; 24.9 \%]$ & $0.14(0.2)$ & $38.34(<0.001)$ & 2.489 \\
\hline Hearing impairments & $9.1 \%$ & $23.2 \%[22.5 \% ; 23.9 \%]$ & $0.14(0.19)$ & $39.7(<0.001)$ & 2.548 \\
\hline $\begin{array}{l}\text { School attending } \\
\text { population }\end{array}$ & $8.1 \%$ & $29.5 \%[28.7 \% ; 30.3 \%]$ & $0.21(0.22)$ & $53.44(<0.001)$ & 3.645 \\
\hline Unemployed population & $6.2 \%$ & $24.3 \%[23.5 \% ; 25 \%]$ & $0.18(0.2)$ & $48.32(<0.001)$ & 3.914 \\
\hline Transportation workers & $5.3 \%$ & $16.8 \%[16.2 \% ; 17.4 \%]$ & $0.12(0.17)$ & $36.44(<0.001)$ & 3.174 \\
\hline Cancer & $4.6 \%$ & $28.3 \%[27.5 \% ; 29 \%]$ & $0.24(0.21)$ & $59.33(<0.001)$ & 6.143 \\
\hline Hospitality workers & $3.7 \%$ & $21.6 \%$ [20.9\%;22.3\%] & $0.18(0.18)$ & $52.77(<0.001)$ & 5.840 \\
\hline Bank/Insurance workers & $3.3 \%$ & $18 \%$ [17.4\%;18.6\%] & $0.15(0.17)$ & $45.96(<0.001)$ & 5.448 \\
\hline Farmworker/Fishermen & $3.0 \%$ & $14.4 \%[13.9 \% ; 14.9 \%]$ & $0.11(0.14)$ & $44(<0.001)$ & 4.788 \\
\hline Transmissible diseases & $1.7 \%$ & $17 \%$ [16.4\%;17.7\%] & $0.15(0.18)$ & $46.69(<0.001)$ & 10.005 \\
\hline Real estate workers & $1.4 \%$ & $13.8 \%[13.3 \% ; 14.4 \%]$ & $0.12(0.15)$ & $43.17(<0.001)$ & 9.881 \\
\hline Military employees & $1.3 \%$ & $16.1 \%[15.5 \% ; 16.8 \%]$ & $0.15(0.17)$ & $46.23(<0.001)$ & 12.423 \\
\hline Genetic diseases & $1.1 \%$ & $13.5 \%[12.9 \% ; 14.1 \%]$ & $0.12(0.16)$ & $42.65(<0.001)$ & 12.260 \\
\hline
\end{tabular}


Table 2. Results of the univariate analyses estimating the magnitude of the primary bias according to the sociodemographic characteristics of the participants and type of condition (alpha value and $\mathbf{9 5 \%} \mathrm{CI}$ ).

\begin{tabular}{lll}
\hline Variable & Alpha value & $\mathbf{9 5 \%} \mathbf{C l}$ \\
\hline Male & 0.56 & {$[0.52 ; 0.6]$} \\
Female & 0.37 & {$[0.34 ; 0.41]$} \\
Middle School & 0.31 & {$[0.28 ; 0.35]$} \\
High School & 0.41 & {$[0.37 ; 0.45]$} \\
Some College and higher & 0.54 & {$[0.5 ; 0.58]$} \\
18 to 39 years old & 0.45 & {$[0.42 ; 0.49]$} \\
40 to 54 years old & 0.43 & {$[0.39 ; 0.47]$} \\
55 years old and older & 0.45 & {$[0.42 ; 0.49]$} \\
Low Numeracy $(<5)$ & 0.25 & {$[0.21 ; 0.28]$} \\
Medium Numeracy $(>=5 \&<8)$ & 0.46 & {$[0.42 ; 0.5]$} \\
High Numeracy $(>=8)$ & 0.63 & {$[0.59 ; 0.68]$} \\
Low SES & 0.38 & {$[0.34 ; 0.42]$} \\
Jobless & 0.42 & {$[0.39 ; 0.46]$} \\
Intermediate SES & 0.54 & {$[0.49 ; 0.59]$} \\
High SES & 0.56 & {$[0.5 ; 0.62]$} \\
Non-health related condition & 0.44 & {$[0.4 ; 0.47]$} \\
Health related condition & 0.46 & {$[0.42 ; 0.49]$} \\
\hline
\end{tabular}


Table 3. Results for the multivariate analyses estimating the magnitude of the primary bias according to the sociodemographic characteristics of the participants and type of condition (alpha value and 95\% CI).

\begin{tabular}{|c|c|c|c|c|c|c|c|c|}
\hline \multirow{2}{*}{ Parameter } & \multicolumn{2}{|c|}{ Estimates } & \multicolumn{2}{|c|}{ Mean error increase } & \multicolumn{2}{|c|}{ Mean absolute error } & \multicolumn{2}{|c|}{ Mean relative error } \\
\hline & & $95 \% \mathrm{Cl}$ & & $95 \% \mathrm{Cl}$ & & $95 \% \mathrm{Cl}$ & & $95 \% \mathrm{Cl}$ \\
\hline Intercept & 1.50 & {$[1.13 ; 1.86]$} & & & & & & \\
\hline Male & REF & & $0 \%$ & & $3.01 \%$ & [2.12\%;4.07\%] & $61.23 \%$ & [41.62\%;85.85\%] \\
\hline Female & -0.67 & {$[-0.85 ;-0.49]$} & $47.9 \%$ & [32.7\%;68.07\%] & $4.47 \%$ & [2.99\%;5.88\%] & $96.58 \%$ & [61.18\%;132.04\%] \\
\hline More than high school & REF & & $0 \%$ & & $3.01 \%$ & [2.12\%;4.07\%] & $61.23 \%$ & [41.62\%;85.85\%] \\
\hline High school diploma & -0.40 & {$[-0.61 ;-0.19]$} & $11.34 \%$ & [6.15\%;17.36\%] & $3.38 \%$ & [2.36\%;4.55\%] & $69.75 \%$ & [46.97\%;97.76\%] \\
\hline Less than high school & -0.64 & {$[-0.87 ;-0.41]$} & $18.71 \%$ & [11.46\%;26.96\%] & $3.56 \%$ & [2.38\%;4.84\%] & $74.17 \%$ & [47.35\%;105.52\%] \\
\hline $\begin{array}{l}\text { Older than } 55 \text { years } \\
\text { old }\end{array}$ & REF & & $0 \%$ & & $3.01 \%$ & [2.12\%;4.07\%] & $61.23 \%$ & [41.62\%;85.85\%] \\
\hline 40 to 54 years old & -0.20 & {$[-0.43 ; 0.03]$} & $5.39 \%$ & [-0.97\%;13.78\%] & $3.26 \%$ & [2.26\%;4.27\%] & $66.89 \%$ & [44.6\%;90.7\%] \\
\hline 18 to 39 years old & -0.32 & {$[-0.55 ;-0.09]$} & $11.61 \%$ & [3.04\%;21.85\%] & $3.31 \%$ & [2.34\%;4.57\%] & $68.16 \%$ & [46.51\%;98.07\%] \\
\hline Intermediate SES & REF & & $0 \%$ & & $3.01 \%$ & [2.12\%;4.07\%] & $61.23 \%$ & [41.62\%;85.85\%] \\
\hline High SES & -0.11 & {$[-0.45 ; 0.23]$} & $1.56 \%$ & {$[-2.13 \% ; 4.54 \%]$} & $3.02 \%$ & [2.19\%;4.17\%] & $61.46 \%$ & [43.09\%;88.24\%] \\
\hline Low SES & -0.26 & {$[-0.54 ; 0.02]$} & $7.58 \%$ & {$[-0.01 \% ; 16.06 \%]$} & $3.28 \%$ & [2.21\%;4.5\%] & $67.36 \%$ & [43.64\%;96.33\%] \\
\hline Jobless & -0.21 & {$[-0.49 ; 0.06]$} & $9.6 \%$ & {$[-2.89 \% ; 24.2 \%]$} & $3.26 \%$ & {$[2.38 \% ; 4.51 \%]$} & $66.9 \%$ & [47.2\%;96.63\%] \\
\hline $\begin{array}{l}\text { Health related } \\
\text { condition }\end{array}$ & REF & & $0 \%$ & & $3.01 \%$ & [2.12\%;4.07\%] & $61.23 \%$ & [41.62\%;85.85\%] \\
\hline $\begin{array}{l}\text { Non-health related } \\
\text { condition }\end{array}$ & -0.08 & {$[-0.25 ; 0.09]$} & $3.68 \%$ & [-3.65\%;11.88\%] & $3.15 \%$ & [2.13\%;4.17\%] & $64.62 \%$ & [42.14\%;88.62\%] \\
\hline High Numeracy $(>=8)$ & REF & & $0 \%$ & & $3.01 \%$ & [2.12\%;4.07\%] & $61.23 \%$ & [41.62\%;85.85\%] \\
\hline $\begin{array}{l}\text { Intermediate } \\
\text { Numeracy }(>=5 \&<8)\end{array}$ & -0.57 & {$[-0.78 ;-0.35]$} & $28.3 \%$ & [17.88\%;39.91\%] & $3.8 \%$ & [2.68\%;5.2\%] & $79.96 \%$ & [54.01\%;114.24\%] \\
\hline Low Numeracy $(<5)$ & -1.42 & {$[-1.67 ;-1.17]$} & $59.29 \%$ & [43.57\%;74.19\%] & $4.73 \%$ & [3.52\%;6.27\%] & $105.43 \%$ & [75.44\%;146.31\%] \\
\hline
\end{tabular}


Figure 1: Univariate analysis stratified on gender (mean perceived prevalence and standard deviation).

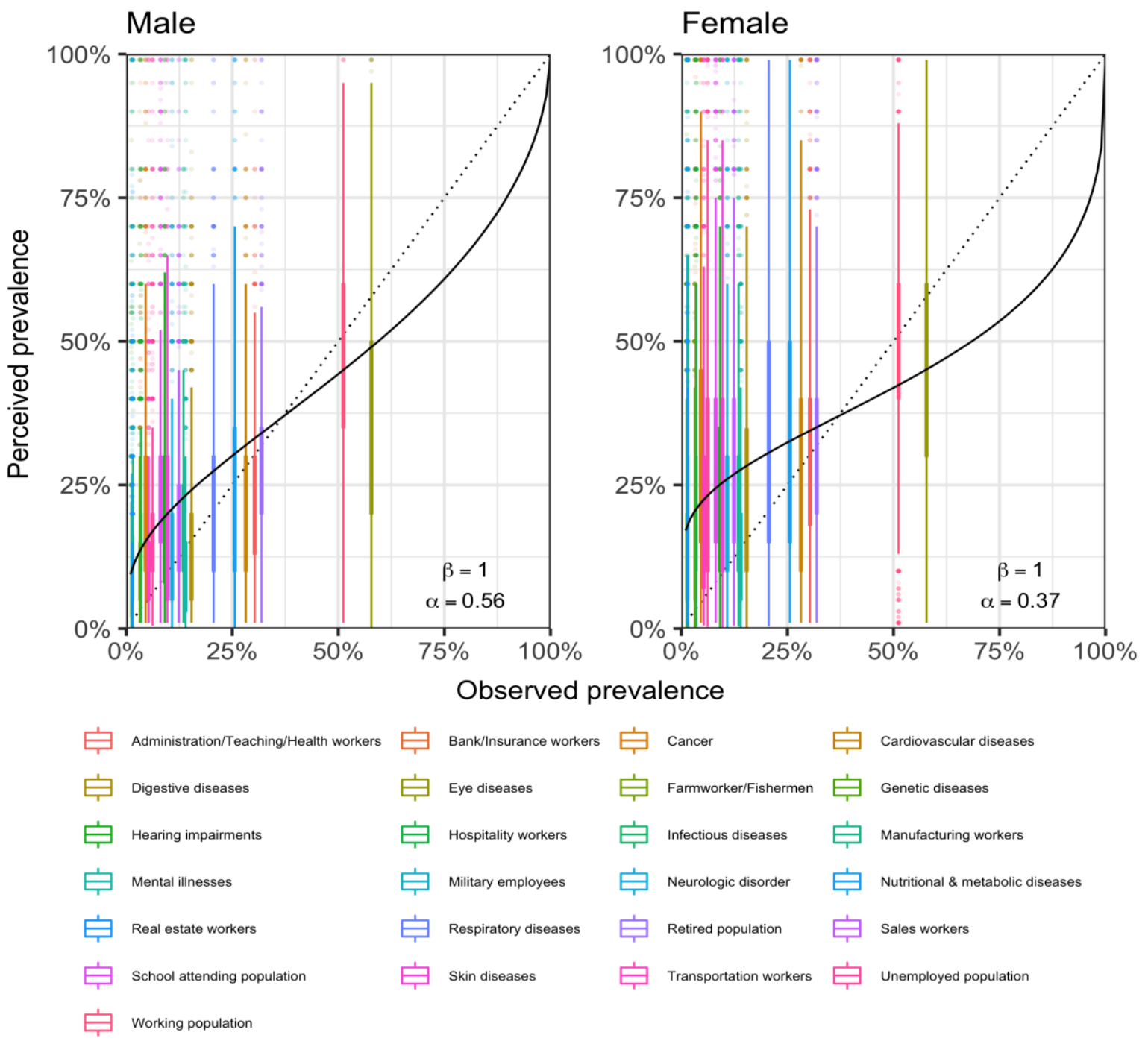


Figure 2: Univariate analysis stratified on level of education (mean perceived prevalence and standard deviation).

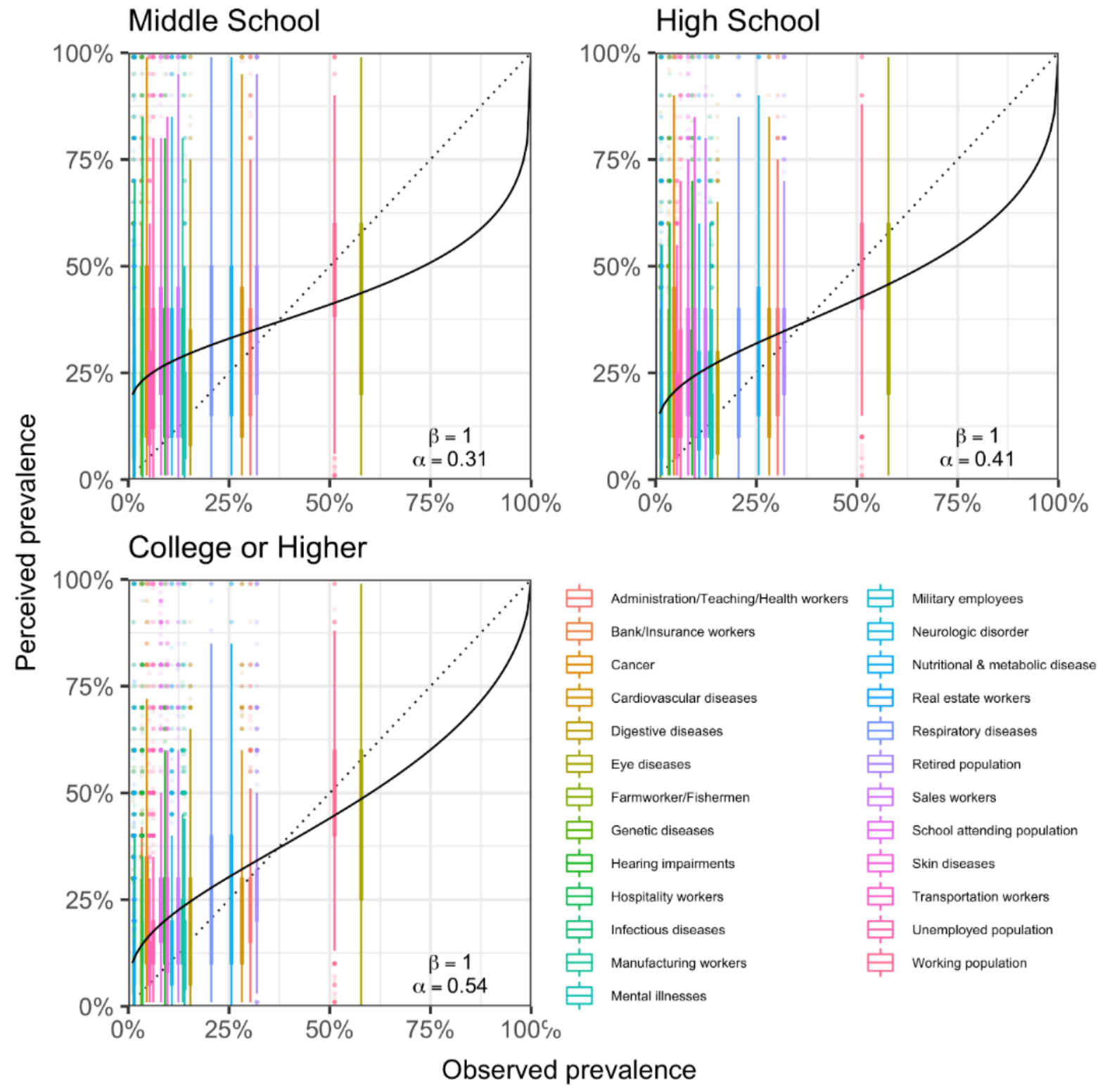


Figure 3. Univariate analysis stratified on numeracy scores (mean perceived prevalence and standard deviation).

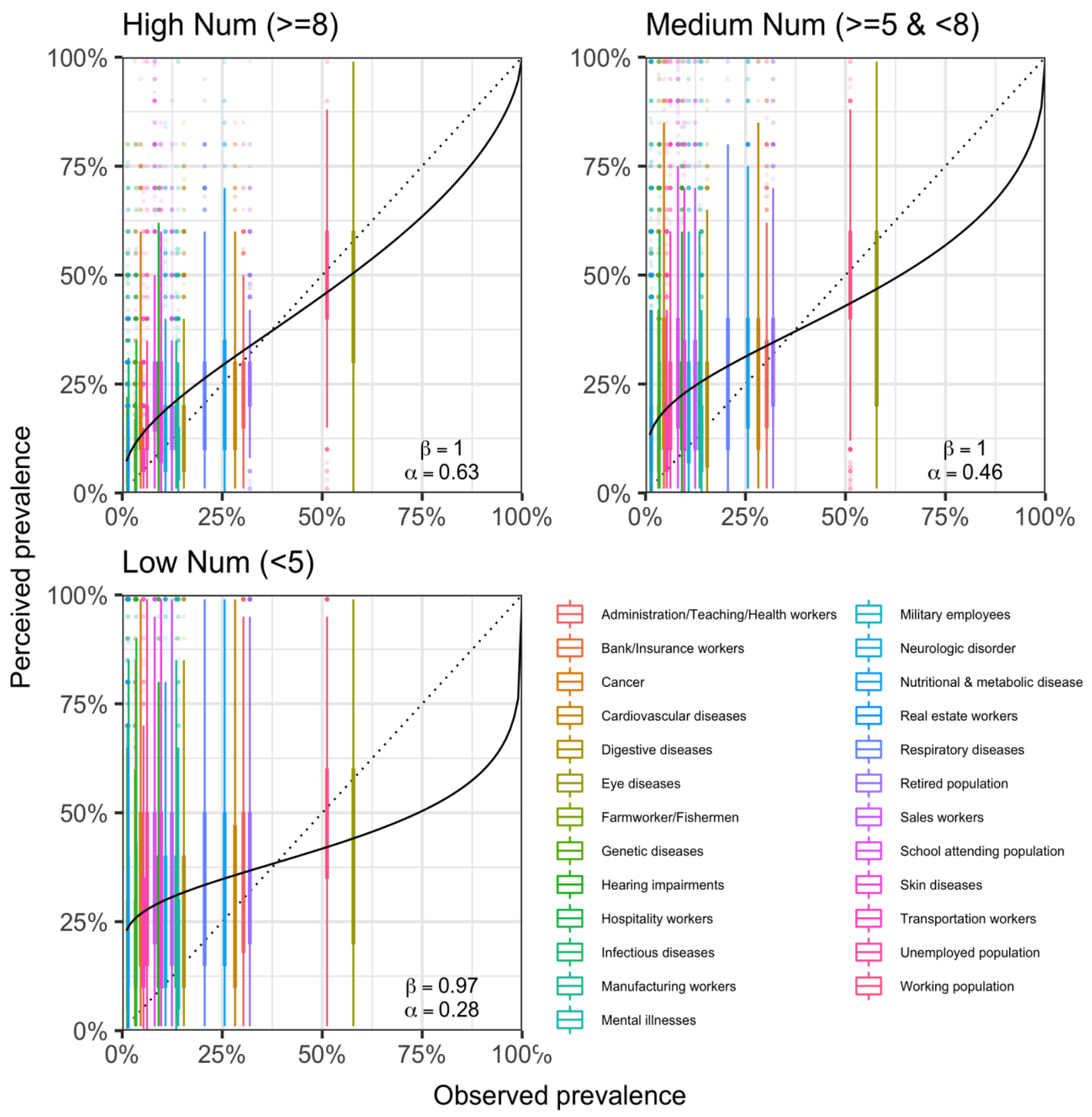


Figure 4. Mean scores across education, and sex on the numeracy scale (0-10)

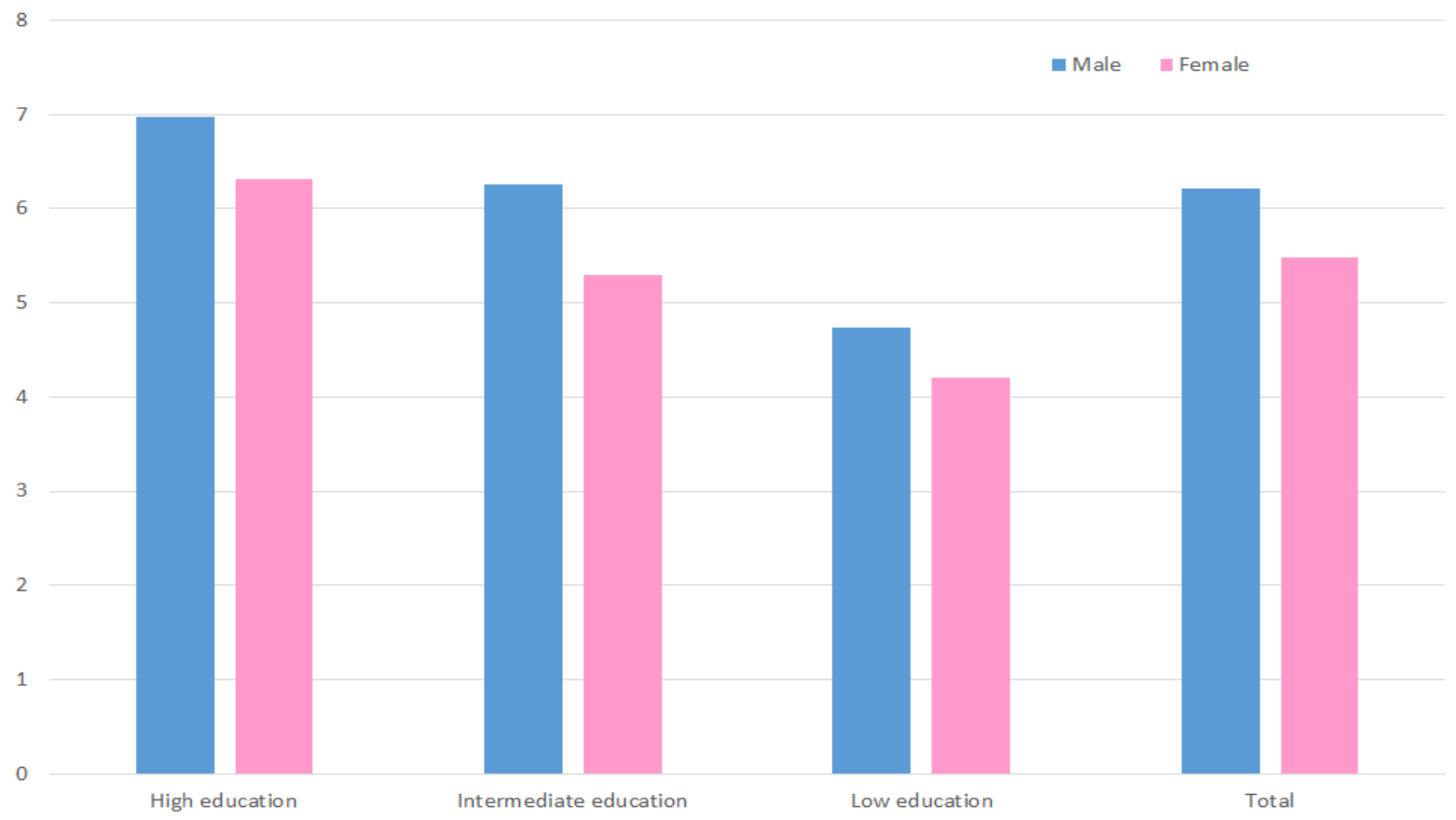


Figure 5. Simple mediation models examining the role of numeracy in sexual and educational differences in the magnitude of the primary bias. Values display unstandardized path coefficients (and standardized path coefficients in parentheses).
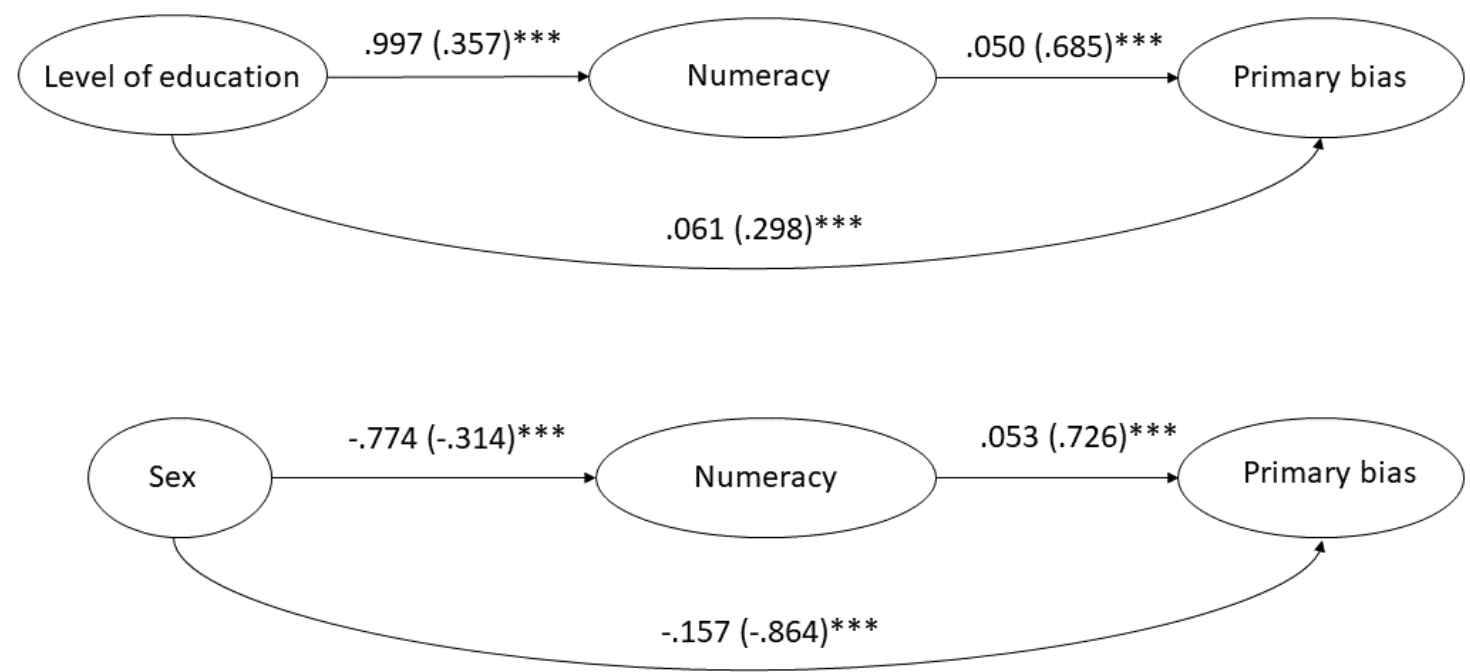

$* * * p<.001$ 